Gesamtbehandlungszeit von zwölf auf 17 Tage. Dem wurde dadurch Rechnung getragen, dass die Gesamtdosis auf 60 Gy in 40 Fraktionen angehoben wurde, im Standardarm auf $66 \mathrm{~Gy}$.

Interessanterweise zeigt die Schwesterstudie des CHART-Protokolls bei Plattenepithelkarzinomen des Kopf-Hals-Bereichs, die 2010 abschließend publiziert wurde und die das CHART-Protokoll (54 Gy in zwölf Tagen) gegen eine Dosis von 66 Gy in 6,5 Wochen bei insgesamt 918 Patienten randomisiert hatte, keinen Unterschied zwischen den Studienarmen [Saunders M et al. Int J Radiat Oncol Biol Phys. 2010;77(1):3-8].

Neben der Interpretation, dass die Verkürzung der Gesamtbehandlungszeit damit 12 Gy an Dosis kompensieren konnte, ist im Licht der CHARTWEL-Ergebnisse auch zu überlegen, ob bei der Standardfraktionierung nicht supralineare Effekte im Dosisbe- reich zwischen 60 und 66 Gy für die Tumorkontrolle zum Tragen kommen.

Ein sehr wichtiges Ergebnis dieser Studie im Einklang mit den zitierten CHART-Regimes ist, dass die Langzeittoxizität trotz erheblicher Dosis-Zeit-Intensivierung nicht erhöht wurde. Auch in diesem Kollektiv starben mehr Patienten am lokalen Progress als an einer Metastasierung, was die Bedeutung der Lokaltherapie auch beim fortgeschrittenen Lungenkarzinom betont.

In diesem Zusammenhang ist die aktuelle Analyse von M. Partridge et al. zu erwähnen [Radiother Oncol. 2011;99(1):6-11], die eine eindeutige Dosis-Wirkungs-Beziehung für die Strahlentherapie des nichtkleinzelligen Lungenkarzinoms im Review unterschiedlichster Studien zeigt und darüberhinaus einen Vorteil für Regimes mit verkürzter Behandlungszeit. Ein weiterer Hinweis dafür, dass der Zeitfaktor in den von uns derzeit verwendeten biologischen Modellen nicht ausreichend abgebildet wird.

Die CHARTWEL-Studie ist auf den ersten Blick eine negative Studie. Der wissenschaftliche Gewinn ist jedoch beträchtlich: Klinische Bestätigung grundlegender strahlenbiologischer Hypothesen, Rationale für verkürzte Behandlungsschemata, Isoeffekt-Beziehungen für Tumorkontrolle und eine Vielzahl von Normalgewebstoxizitäten.

Der derzeitige Standard für lokal fortgeschrittene Stadien bleibt die definitive simultane Radiochemotherapie mit Dosen um 66 Gy.

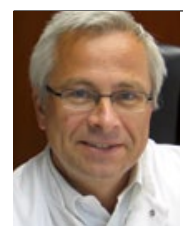

Prof. Dr. Michael Flentje

Klinik und Poliklinik für Strahlentherapie, Universitätsklinikum Würzburg flentje_m@klinik.uniwuerzburg.de

\section{Verblüffendes Studienergebnis Lungenkrebs: Überlebensvorteil für Diabetiker}

\section{Diabetes mellitus ist eine häufige Zusatzerkrankung von Patienten mit Bronchialkarzinom. Doch während die Stoffwechselkrankheit bei anderen Tumorarten die Prognose verschlechtert, scheint sie beim Lungenkrebs mit verlängerten Überlebenszeiten einherzugehen.}

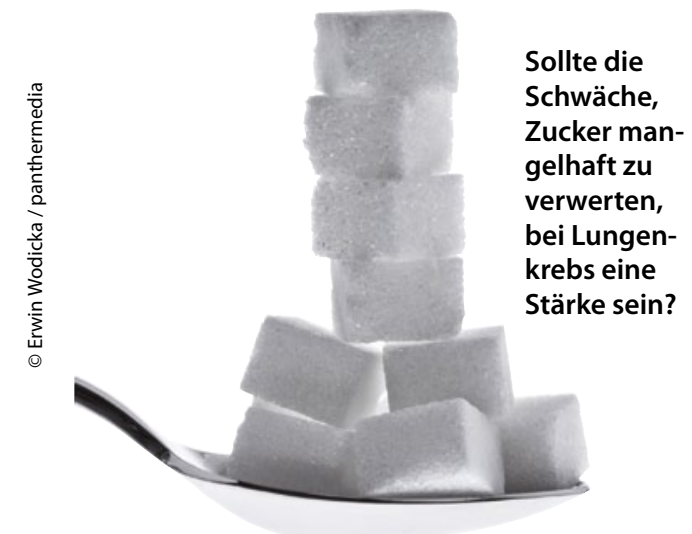

_ orscher um Peter Hatlen von der Universität Trondheim hatten die Daten von 1.677 Lungenkrebs-Patienten analysiert, von denen 77 zugleich an Diabetes mellitus litten. Eingerechnet wurden die Angaben zu Alter, Geschlecht, Tumorhistologie und -stadium. Die Diabetes-Diagnose erwies sich dabei als Überlebensvorteil, die mediane Überlebenszeit war bei den Diabetikern mit zehn Monaten deutlich länger als bei den Nicht-Diabetikern, die median sechs Monate überlebten $(\mathrm{p}=0,005)$.

Ein Jahr nach der Krebsdiagnose waren noch $43 \%$ der Diabetiker am Leben, verglichen mit $28 \%$ der Patienten ohne Diabetes; und auch nach zwei (19 vs. $11 \%$ ) und drei Jahren (3 vs. $1 \%$ ) waren die Überlebensraten der Diabetiker höher $(p=0,005)$. Der Diabetes erwies sich als unabhängiger Prädiktor für ein verlängertes Überleben (Hazard Ratio 0,55). Die Gründe dafür, weshalb lungenkrebskranke Diabetiker eine bessere Prognose aufweisen als Nicht-Diabetiker mit Bronchialkarzinomen, sind unklar. Zwar hatten Diabetiker seltener Metastasen als NichtDiabetiker, doch in den Berechnungen war das Krankheitsstadium berücksichtigt worden. Zudem ließ sich der Überlebensvorteil gerade in fortgeschrittenen Stadien des Krebsleidens demonstrieren. Der günstige Einfluss des Diabetes lässt sich des- halb auch nicht mit der besseren medizinischen Betreuung von Diabetikern bzw. häufigeren Kontrolluntersuchungen erklären, die zu früherer Diagnose und besserer Prognose hätten führen können.

Fazit: Die Diagnose eines Diabetes mellitus ist laut den Autoren kein Grund, Lungenkrebs-Patienten die Standardtherapie vorzuenthalten: „Der Nutzen fürs Überleben ist möglicherweise klinisch relevant und sollte in künftigen Studien beobachtet werden." Robert Bublak

Hatlen $P$ et al. Prolonged survival in patients with lung cancer with diabetes mellitus.

JThorac Oncol. 2011;6(11):1810-7.

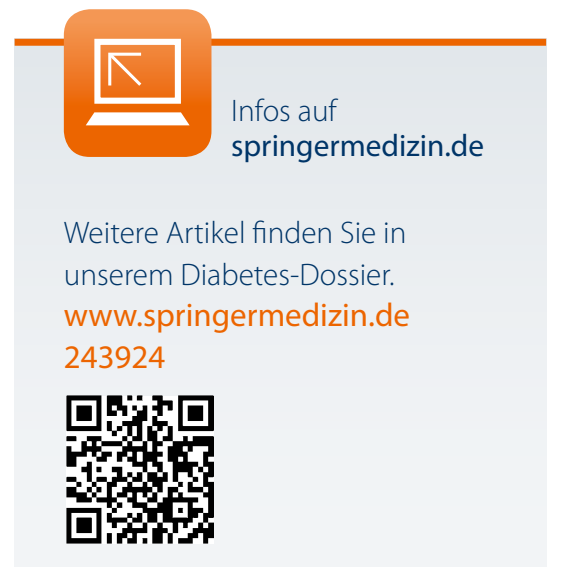

